\title{
ARTICLE
}

Received 23 Sep 2015 | Accepted 2 Nov 2015 | Published 7 Dec $2015 \quad$ DOl: 10.1038/ncomms10098 OPEN

\section{Arp2/3-mediated F-actin formation controls regulated exocytosis in vivo}

Duy T. Tran ${ }^{1}$, Andrius Masedunskas ${ }^{2,3}$, Roberto Weigert ${ }^{2} \&$ Kelly G. Ten Hagen ${ }^{1}$

The actin cytoskeleton plays crucial roles in many cellular processes, including regulated secretion. However, the mechanisms controlling F-actin dynamics in this process are largely unknown. Through 3D time-lapse imaging in a secreting organ, we show that F-actin is actively disassembled along the apical plasma membrane at the site of secretory vesicle fusion and re-assembled directionally on vesicle membranes. Moreover, we show that fusion pore formation and $\mathrm{PIP}_{2}$ redistribution precedes actin and myosin recruitment to secretory vesicle membranes. Finally, we show essential roles for the branched actin nucleators Arp2/3and WASp in the process of secretory cargo expulsion and integration of vesicular membranes with the apical plasma membrane. Our results highlight previously unknown roles for branched actin in exocytosis and provide a genetically tractable system to image the temporal and spatial dynamics of polarized secretion in vivo.

\footnotetext{
${ }^{1}$ Developmental Glycobiology Section, NIDCR, National Institutes of Health, Building 30, Room 426, 30 Convent Drive, MSC 4370, Bethesda, Maryland 20892, USA. ${ }^{2}$ Intracellular Membrane Trafficking Section, NIDCR, National Institutes of Health, 30 Convent Drive, Bethesda, Maryland 20892, USA.

${ }^{3}$ Oncology Research Unit, School of Medical Sciences, University of New South Wales, Wallace Wurth Building East (C27), Room 227, Sydney, New South Wales 2052, Australia. Correspondence and requests for materials should be addressed to K.G.T.H. (email: Kelly.Tenhagen@nih.gov).
} 
$\mathrm{R}$ egulated exocytosis is a fundamental cellular process that involves the release of stored secretory cargo from membranous vesicles into the extracellular space. This type of secretion involves the formation of secretory vesicles and their subsequent docking and fusion with the plasma membrane $(\mathrm{PM})$ in response to an external stimulus. Various tissues have different modes of vesicle formation and release, likely due to the different physical and functional properties of the secreted cargo as well as the size/geometry of the secretory vesicles. Regulated secretion is used by specialized tissues to produce and secrete bioactive molecules that mediate diverse functions including neurotransmission, immunity, reproduction and digestion (reviewed in refs 1-3).

Recent studies have highlighted essential, yet often disparate roles for the actin cytoskeleton in regulated exocytosis and in compensatory endocytosis. For example, actin along the cortex of cells acts as a physical barrier to prevent premature fusion of granules with the $\mathrm{PM}^{4-6}$. In Xenopus eggs, where secretory vesicles never integrate into the PM but are maintained as shells, actin and myosin recruitment enable vesicles to be retrieved via compensatory endocytosis ${ }^{7-10}$. Other studies have demonstrated that F-actin is required for proper vesicle compression and expulsion of cargo ${ }^{1-14}$ and to regulate the expansion of the fusion pore ${ }^{15}$. In addition, intravital imaging in rodent salivary glands (SGs) has shown that F-actin is required to regulate both the stabilization and collapse of secretory vesicles after fusion with the $\mathrm{PM}$ in vivo ${ }^{16}$. However, the molecular machinery controlling the formation of specific F-actin structures and the spatial kinetics of actin assembly in the process of cargo expulsion and membrane integration has not been fully defined ${ }^{3}$.

Actin exists as monomeric units (G-actin) and in a variety of filaments that can be linear, branched or bundled (F-actin). Linear actin filaments are initiated by the formin-family of actin nucleators/elongation factors that are activated by the concerted action of small Rho-GTPases and phosphoinositides ${ }^{17-19}$. Rho and formin influence actin coat formation on lamellar bodies during surfactant secretion ${ }^{12,13}$. Branched actin filaments, which are essential for cell polarity and migration ${ }^{20,21}$, are formed through the coordinated action of the Actin-related protein 2/Actin-related protein 3 (Arp2/3) complex and a variety of nucleation-promoting factors (NPFs), such as WASp, Wash, Whamy and suppressor of cAR (SCAR) $)^{20,22-24}$. Arp2/3 present on linear actin filaments is subsequently activated by NPFs, which bind Arp2/3 and induce a structural change. Branched actin is then formed at the site of Arp2/3 attachment at a $70^{\circ}$ angle from the existing actin filament ${ }^{25,26}$. Actin nucleators influence glucose-induced biphasic insulin secretion, but their specific effects on actin remodelling in this process are unclear ${ }^{27}$. The role of branched actin assembly and structure is less well understood in other forms of secretion that involve large secretory vesicles containing bulky cargo ${ }^{3}$.

The factors that govern regulated secretion have been challenging to decipher, as many mammalian cells lose polarity and secretory capacity once excised and cultured ex vivo ${ }^{28}$. Additionally, in many systems, the small size of vesicular structures and the lack of markers for cargo impede the ability to image actin dynamics, secretory vesicles and secretion with sufficient resolution. Furthermore, limited genetic tools in many systems have restricted the ability to interrogate the function of specific genes involved in actin formation in vivo.

To investigate the role of actin dynamics and branched actin in secretion, we performed high-resolution, real-time imaging at single-granule resolution in Drosophila SGs, which are amenable to genetic manipulation and contain large secretory granules (3$8 \mu \mathrm{M}$ in diameter) filled with high-molecular-weight cargo. Using Drosophila lines expressing fluorescently-labelled molecules, we are able to directly image secretory vesicle cargo, apical membrane dynamics and actin and myosin recruitment in real time to define the temporal and spatial sequence of events occurring during regulated exocytosis. By performing 4-dimensional (4D) imaging of actin dynamics during secretion, we show rearrangement of cortical actin at the point of vesicle fusion followed by coordinated recruitment of actin to the fused vesicle from the PM. We further demonstrate that fusion pore formation and redistribution of the phosphoinositide, $\mathrm{PIP}_{2}$, occurs before actin recruitment. By imaging cargo directly, we show that fusion pore formation and cargo secretion are temporally distinct events, with the latter being dependent on actin for completion. Finally, using in vivo RNA interference (RNAi), we identify essential roles for the branched actin nucleators Arp2, Arp3 and their activator, WASp, in mediating cargo expulsion and secretory granule membrane integration with the PM. This study demonstrates a previously unknown role for branched actin nucleators in exocytosis and offers a high-resolution, genetically tractable platform for interrogating the role of any factor involved in actin dynamics and regulated secretion in a living organ in real time.

\section{Results}

Spatial and temporal dynamics of regulated secretion. The Drosophila larval salivary system consists of two glands comprised of secretory epithelial cells that connect to a common duct (Fig. 1a). Secretory cells are filled with large secretory granules (3-8 $\mu \mathrm{M}$ in diameter) containing high molecular weight, highly glycosylated mucins (salivary gland secretion proteins or sgs proteins $)^{29,30}$. Regulated secretion in Drosophila SGs begins with a small pulse of the steroid hormone 20-hydroxyecdysone (20E) during early third instar larval stage. This stimulus induces the expression of the sgs genes that encode the cargo that will be packaged into secretory vesicles; then, a second pulse of $20 \mathrm{E}$ during late third instar instructs these newly formed secretory granules to fuse with the apical PM to begin secretion ${ }^{31-33}$. To image secretion events in real time, we took advantage of Drosophila lines expressing fluorescent markers for secretory cargo (Sgs3-GFP) ${ }^{32}$, F-actin (Lifeact-Ruby, Lifeact-RFP or Lifeact-GFP) ${ }^{34,35}$ and the apical membrane (Myr-tdTomato) $)^{36}$ SGs from Sgs3-GFP-expressing larvae were harvested during late third instar and cultured ex vivo. Cultured SGs maintain their polarity and respond to the exogenous addition of 20E, which triggers fusion of the secretory vesicles with the apical PM and release of their contents into the lumen (Fig. 1b). As secretion proceeds, the diameter of the lumen expands as it fills with the Sgs3-GFP cargo (Fig. 1b and Supplementary Movie 1), similar to what is seen in vivo (Supplementary Fig. 1a). (Real-time videos of all still images shown throughout this paper are in the Supplementary Movies section.) Imaging the dynamics of individual secretory granules using Drosophila lines expressing Sgs3-GFP cargo and the apical PM marker Myr-tdTomato revealed apical membrane deformations at the point of vesicle fusion (Fig. 1c). In addition, individual secretory vesicles undergo a slight expansion after fusion with the apical PM, likely due to hydration-related expansion of the mucin cargo. Real-time imaging of secretory cargo revealed that secretion proceeds with complete expulsion of cargo followed by integration of the vesicle membranes with the PM (Fig. 1c and Supplementary Movie 2).

We next imaged the dynamics of actin recruitment to individual secretory granules using Drosophila lines expressing both Sgs3-GFP and Lifeact-Ruby. Secretory vesicles in proximity to the apical membrane are rapidly coated with actin, which precedes vesicle compression and membrane integration (Fig. 1d 
a

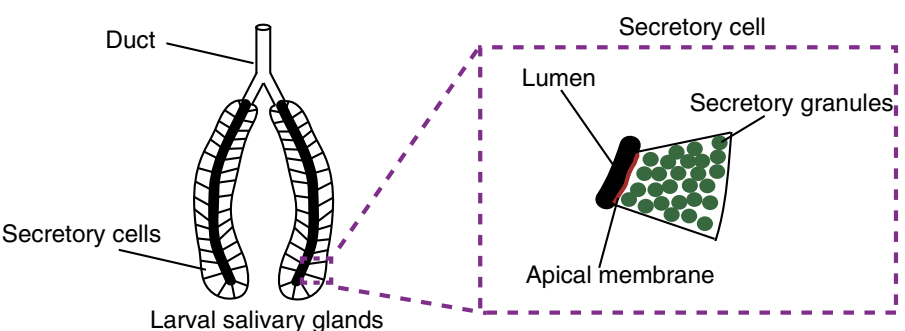

b

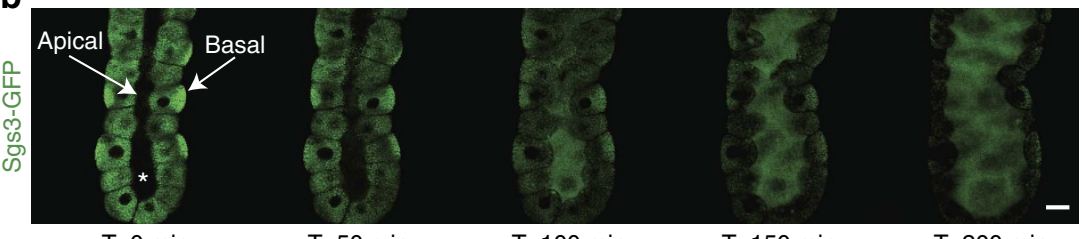

C

Sgs3-GFP Myr-tdTomato

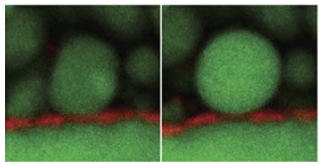

T: $0 \mathrm{~s}$

T: $25 \mathrm{~s}$

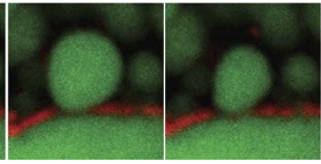

T: $50 \mathrm{~s}$

T: $75 \mathrm{~s}$

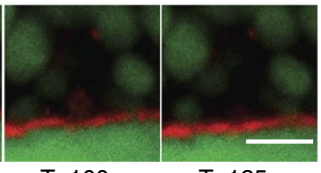

$\mathrm{T}: 100 \mathrm{~s}$

$\mathrm{T}: 125 \mathrm{~s}$

d

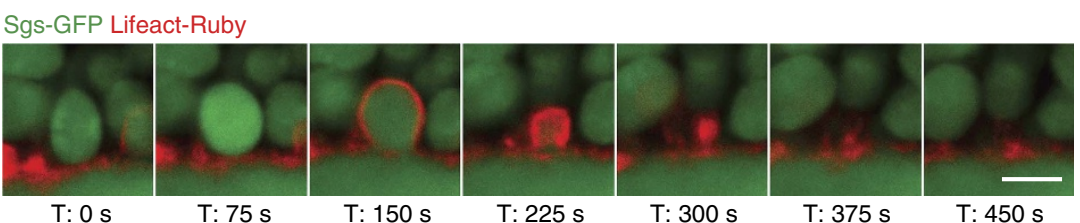

e

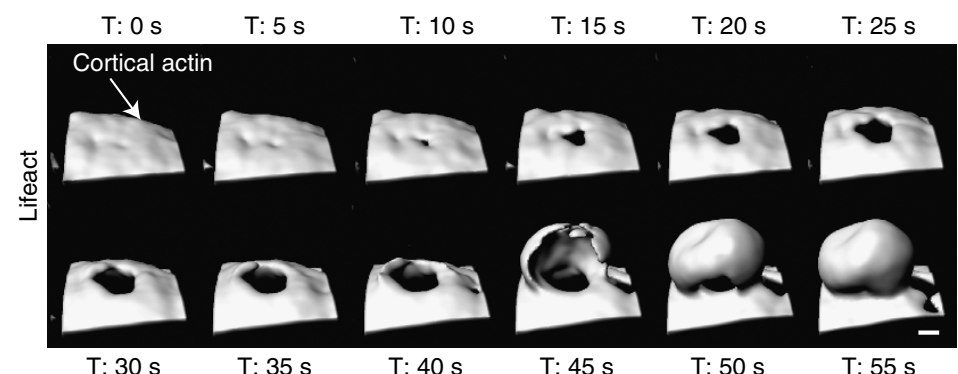

Figure 1 | Real-time imaging of actin dynamics during regulated exocytosis. (a) Schematic diagram of third instar larval salivary glands (SGs). Inset depicts a single secretory cell. (b) Third instar larval SGs expressing Sgs3-GFP cargo (green) were imaged in real time after induction of secretion by the addition of 20E. Sgs3-GFP cargo in secretory vesicles is secreted into the lumen over time, leading to lumenal expansion. The distal tip of the gland is at the bottom of the image. Apical and basal surfaces are labelled. The SG lumen is denoted by a *. A representative time series from four independent experiments is shown. Scale bar, $50 \mu \mathrm{m}$. (c) Individual granules from SGs expressing Sgs3-GFP (green) and Myr-tdTomato (red; an apical PM marker) were imaged to show vesicle fusion with the PM and deformation of the PM at the site of fusion. After cargo expulsion, integration of granular membrane with the PM is seen. A representative time series from six independent experiments is shown. Scale bar, $5 \mu \mathrm{m}$. (d) Granule fusion in glands expressing Sgs3-GFP (green) and Lifeact-Ruby (red) shows actin recruitment to the fusing secretory granule, followed by expulsion of green secretory cargo into the lumen and integration of the granule into the PM. A representative time series from six independent experiments is shown. Scale bar, $5 \mu \mathrm{m}$. (e) Surface reconstruction of 4D Lifeact-GFP (white) imaging during granule secretion showing actin clearance at the apical PM followed by actin recruitment from the PM to the secretory vesicle. A representative time series from nine granule reconstructions over three independent experiments is shown. Scale bar, $3 \mu \mathrm{m}$. Complete movies for each time series can be found in the Supplementary Movie section.

and Supplementary Movie 3). To image actin recruitment around the entirety of the vesicle, we performed three-dimensional imaging in real time (4D imaging) in Drosophila lines expressing Lifeact-GFP. Interestingly, $4 \mathrm{D}$ reconstruction of actin dynamics showed that cortical actin present along the apical membrane is cleared or disrupted at the site of vesicle fusion, forming a small hole that continues to expand in size (Fig. 1e and Supplementary
Fig. 1b,c and Supplementary Movies 4, and 5). This actin clearance is followed by actin recruitment to the secretory vesicle, originating at the PM (Fig. 1e and Supplementary Fig. 1b, $c$ and Supplementary Movie 4, and 5). These 4D reconstructions demonstrate dynamic actin clearance at the PM and subsequent directional actin recruitment to the secretory vesicle from the PM during SG secretion. Taken together, we show secretion of 
mucinous cargo at single granule resolution that involves dynamic rearrangement of both the cortical actin and the apical membrane at the site of secretory vesicle fusion. This process culminates in complete expulsion of cargo and integration of the granular membrane with the PM.

We next addressed the temporal order of membrane fusion (fusion pore formation) and actin recruitment to the secretory vesicles. By infusing a small molecular weight (MW) fluorescent dextran (A647-10 kDa dextran, stoke radius $\sim 2 \mathrm{~nm}$ (ref. 15)) into the lumens of SGs expressing Lifeact-GFP, we demonstrate that the fusion pore between the vesicle and the PMs forms before F-actin recruitment to the secretory vesicle (Fig. 2a, d and Supplementary Fig. 2a). Indeed, dextran diffusion from the SG lumen into the vesicle was detected before Lifeact-GFP (F-actin) on vesicle membranes (Supplementary Movie 6). The average time difference in the detection of dextran relative to actin was $57 \pm 18 \mathrm{~s}$ ( $n=11 ; \pm$ refers to standard deviation). In addition, we did not see any evidence for compound exocytosis (for example, the sequential fusion of vesicles with one another at the PM), consistent with what was previously reported in mammalian SGs ${ }^{16}$.

Membrane mixing and the redistribution of phosphoinositides are known to regulate actin polymerization and membrane fusion events in other systems ${ }^{37}$. In particular, the membrane phosphoinositide $\mathrm{PI}(4,5) \mathrm{P}_{2}\left(\mathrm{PIP}_{2}\right)$ is known to stimulate actin polymerization by regulating the activity of actin nucleators ${ }^{38,39}$. Therefore, we next examined whether redistribution of $\mathrm{PIP}_{2}$ occurs during SG secretion by using Drosophila lines expressing both the $\mathrm{PIP}_{2}$ reporter PLC $\delta$-PH-EGFP ${ }^{40}$ and Lifeact-RFP. Indeed, evidence for membrane mixing via $\mathrm{PIP}_{2}$ movement from the apical PM to secretory vesicle membranes is seen (Fig. 2b,e and Supplementary Movie 7). Interestingly, $\mathrm{PIP}_{2}$ recruitment precedes F-actin recruitment (by $51 \pm 19 \mathrm{~s}(n=12)$ ), consistent with a role for $\mathrm{PIP}_{2}$ in $\mathrm{F}$-actin formation along secretory vesicle membranes (Fig. 2b,e and Supplementary Fig. 2b and Supplementary Movie 7).

In rodent exocrine glands, it was shown that the actin motor non-muscle myosin II is recruited onto the secretory granules, but the temporal sequence of events is not known ${ }^{16}$. To determine the kinetics of myosin recruitment relative to actin recruitment, we performed time-lapse imaging in flies co-expressing the myosin marker non-muscle myosin II (Zip-GFP) $)^{41,42}$ and Lifeact-Ruby. As shown in Fig. 2c,f and Supplementary Fig. 3a and Supplementary Movie 8, myosin is detected on fused vesicles after F-actin. The average time difference in the detection of actin relative to myosin was $25 \pm 13 \mathrm{~s}(n=11)$. Altogether, our data show that secretion in Drosophila SGs begins with fusion pore formation and redistribution of $\mathrm{PIP}_{2}$, followed by directional actin recruitment to secretory vesicle membranes and, finally, myosin recruitment. Moreover, secretion of the cargo is followed by complete membrane integration of secretory vesicles with the apical PM. Therefore, our real-time imaging system provides spatial and temporal resolution to image dynamic events occurring on the membranes of individual secretory granules in a living organ.

Arp2/3 F-actin formation is required for secretion. The structural complexities associated with large, highly glycosylated cargo such as mucins ${ }^{43,44}$, may require actomyosin contractile forces for cargo expulsion and membrane integration. We therefore examined the role of F-actin in SG secretion. We had previously shown that pharmacological disruption of the actin cytoskeleton by latrunculin A (LA) or cytochalasin D (CD) treatment impairs the gradual collapse of the secretory granules in rodent SGs in vivo ${ }^{16}$. Likewise, treatment of Drosophila SGs with
LA or CD also resulted in disrupted secretion (Fig. 3a,b, Supplementary Fig. 5a,c and Supplementary Movies 9-12). Treatment with either drug severely inhibited vesicle collapse as fused vesicles expanded dramatically in size, underwent compound fusion and failed to undergo membrane integration (Fig. 3a,b, Supplementary Fig. 5a,c and Supplementary Movies 9-12). The efficacy of each treatment on the actin cytoskeleton was assessed by examining both Lifeact-Ruby in real time and by staining fixed glands with phalloidin (Fig. 3c, Supplementary Figs $4 \mathrm{a}, \mathrm{b}$ and $5 \mathrm{~b}$ and Supplementary Movies 13-16). Taken together, these results demonstrate a requirement for F-actin in regulated secretion in this system and highlight its role in vesicle collapse and the prevention of compound fusion.

Conventional light microscopy cannot resolve the organization of actin filaments on the surface of the membranes of the secretory granules in Drosophila SGs. Nonetheless, based on the fact that F-actin on the granules prevents compound exocytosis, we speculated that filaments may be tightly organized around the membranes, possibly by employing extensive branching. Branched actin is nucleated from linear filaments via the Arp2/3 complex and NPFs. To determine which of these proteins may be involved in regulated secretion within Drosophila SGs, we performed immunofluorescent staining on actively secreting glands. Arp3, WASp, Whamy and SCAR (but not Wash) each co-localized with phalloidin on granules that had fused with the apical PM, suggesting a role for these factors in secretion (Fig. 4a). We next examined the temporal order of recruitment of the Arp3 complex onto the secreting granules by creating a Drosophila line expressing both GFP-labelled Arp3 (Arp3-GFP) and Lifeact-Ruby. Real-time imaging of Lifeact-Ruby and Arp3-GFP during secretion revealed that F-actin is seen on granules before Arp3 recruitment (Fig. 4b,c, Supplementary Fig. $3 \mathrm{~b}$ and Supplementary Movie 17). The average time difference in the detection of F-actin relative to Arp3 was $29 \pm 14 \mathrm{~s}(n=13)$. As the Arp $2 / 3$ complex is known to bind linear actin and is required for branched actin formation, our results suggest a model where fused vesicles are initially coated with linear actin, which is detected by Lifeact, and then subsequently bound by Arp2/3 to form a branched actin network.

To investigate whether branched actin is required for regulated exocytosis, we next performed in vivo knockdown of components of the Arp2/3 complex in flies expressing Sgs3-GFP or Lifeact-Ruby. RNAi to Arp3 substantially decreased Arp3 expression and resulted in granules that fused with the apical membrane, but failed to secrete cargo and failed to undergo membrane integration (Fig. 5a, Supplementary Figs 6a,b and 7a and Supplementary Movies 18 and 19). Upon loss of Arp3, fused granules continued to enlarge, similar to what was observed in SGs treated with LA. However, unlike LA treatment, no evidence of compound exocytosis was seen, suggesting that linear actin still present on granules may function to block compound fusion. Interestingly, some F-actin (likely linear actin) was still detected on fused granules in the Arp3 RNAi background, although no longer in a homogenous distribution (Supplementary Fig. 6c, d and Supplementary Movies 20 and 21). Likewise, RNAi to Arp2 (which forms a functional complex with Arp3) phenocopied the knockdown of $A r p 3$, adding further support for the role of Arp2/ 3-mediated branched actin in proper cargo expulsion and membrane integration (Fig. 5b and Supplementary Figs 6a, b and $7 \mathrm{~b}$ and Supplementary Movies 22 and 23). We next examined the role of the Arp $2 / 3$ activator, WASp. RNAi to WASp, an NPF that can activate Arp2/3, also resulted in granules that grew larger in size, failed to expel cargo and failed to undergo membrane integration; these granules also maintained limited and unevenly distributed staining for F-actin (likely detecting linear actin) and did not show evidence of compound fusion (Fig. $5 \mathrm{c}$ and Supplementary Figs $6 a, b, c, e$ and $7 c$ and Supplementary 
a

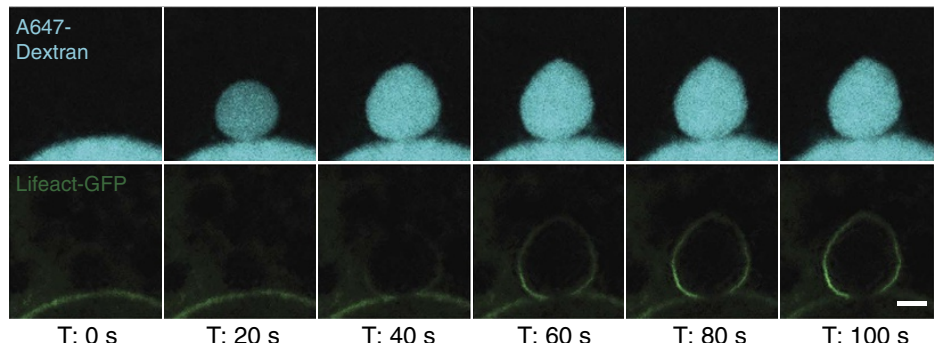

b

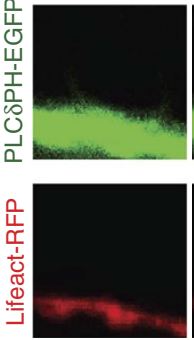

$\mathrm{T}: 0 \mathrm{~s}$
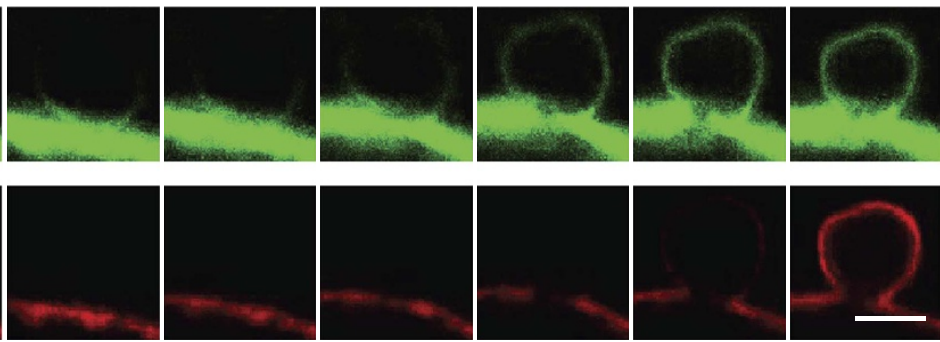

$\mathrm{T}: 25 \mathrm{~s}$
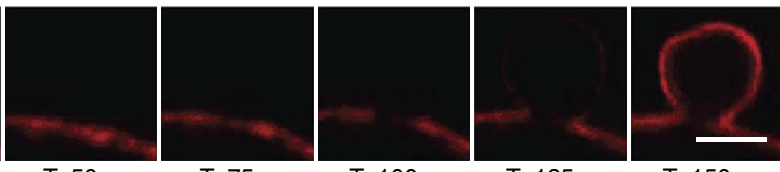

$\mathrm{T}: 125 \mathrm{~s}$

$\mathrm{T}: 150 \mathrm{~s}$

C
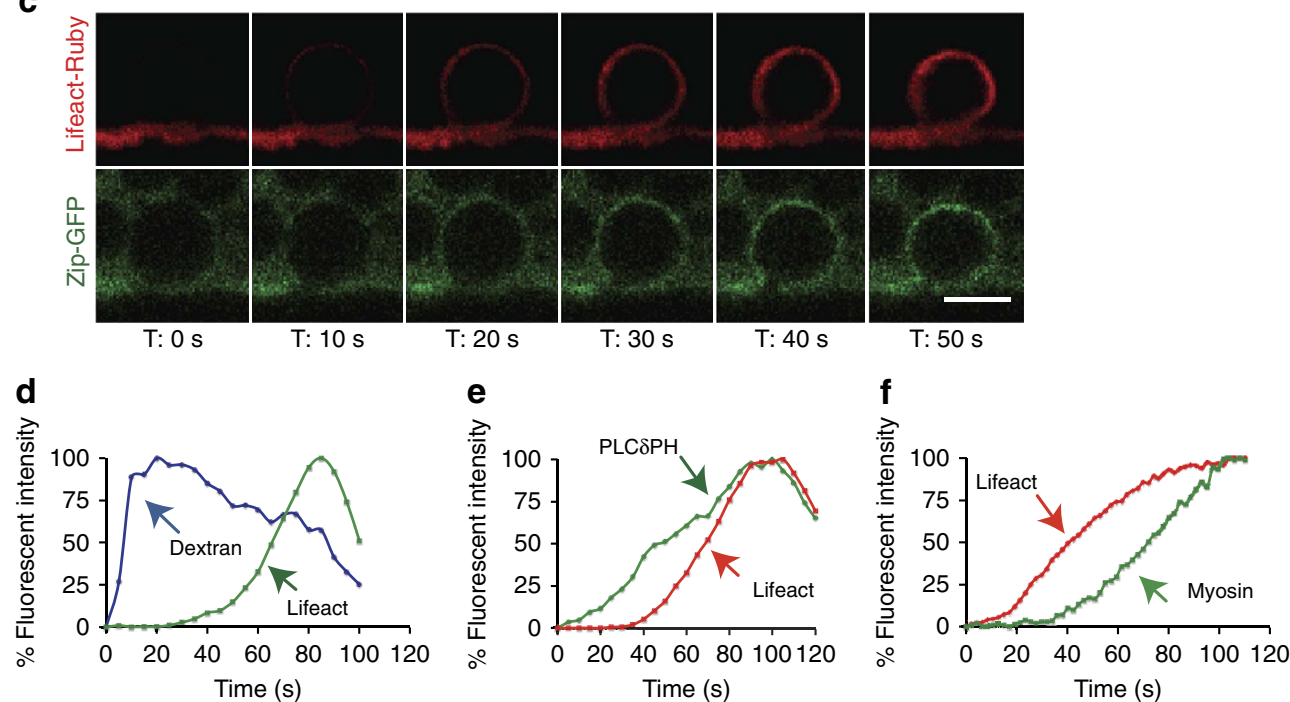

Figure 2 | Fusion pore formation and membrane mixing occur before actin and myosin recruitment on individual secretory granules. (a) The lumen of glands expressing Lifeact-GFP (green) was infused with A647-dextran (cyan) to image fusion pore formation between the granule and the apical PM. Realtime imaging shows fusion pore formation (via diffusion of dextran from the SG lumen into the granules) before actin recruitment to the granule. A representative time series from five independent experiments is shown. Scale bar, $5 \mu \mathrm{m}$. (b) SGs expressing the PIP 2 reporter, PLC $\delta \mathrm{PH}$-EGFP (green) and Lifeact-RFP (red) were imaged in real time to demonstrate that diffusion of PIP 2 from the apical membrane to the fused vesicle membrane occurs before actin recruitment. A representative time series from four independent experiments is shown. Scale bar, $5 \mu$ m. (c) Glands expressing Lifeact-Ruby (red) and Zip-GFP (green; non-muscle myosin II) were imaged in real time to show that actin recruitment to fused granules occurs before myosin recruitment. A representative time series from eight independent experiments is shown. Scale bar, $5 \mu$ m. (d) Quantification of Lifeact recruitment relative to time of dextran entry into fused granules. (e) Quantification of PLC $\delta \mathrm{PH}$ appearance relative to Lifeact recruitment to fused granules. (f) Quantification of Lifeact recruitment relative to myosin recruitment to fused granules. Representative graphs plotting percent fluorescent intensity of each marker as a function of time for individual secretory events are shown. Quantification was performed on 11 individual secretory events over two independent experiments, 12 individual secretory events over two independent experiments and 11 individual secretory events over four independent experiments, respectively. Additional graphs are shown in Supplementary Figs 2a, b and 3a. Complete movies for each time series can be found in the Supplementary Movie section.

Movies 24-27). Finally, large, expanded granules could also be seen in the SGs in the intact pupae in Arp3 RNAi, Arp2 RNAi or WASp RNAi backgrounds (Supplementary Fig. 8), again indicating that our ex vivo SG system faithfully recapitulates what is occurring in vivo. Taken together, our studies demonstrate an essential role for the branched actin nucleators Arp2, Arp3 and WASp in secretory cargo expulsion and membrane integration during regulated exocytosis in vivo (Fig. $5 \mathrm{~d}$ ).

\section{Discussion}

Here, we show temporal and spatial dynamics of F-actin formation during regulated exocytosis and define essential roles for the branched actin nucleators Arp2, Arp3 and WASp in cargo secretion and membrane integration (Fig. 5d). By imaging actin dynamics in four dimensions at single granule resolution in a secreting organ, we demonstrate that actin along the PM is dynamically cleared at the point of vesicle fusion. This 
a

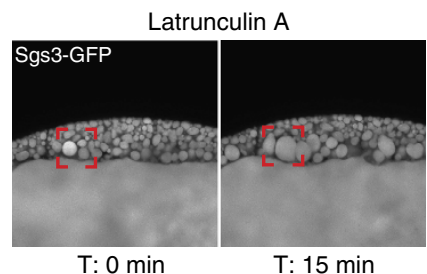

b
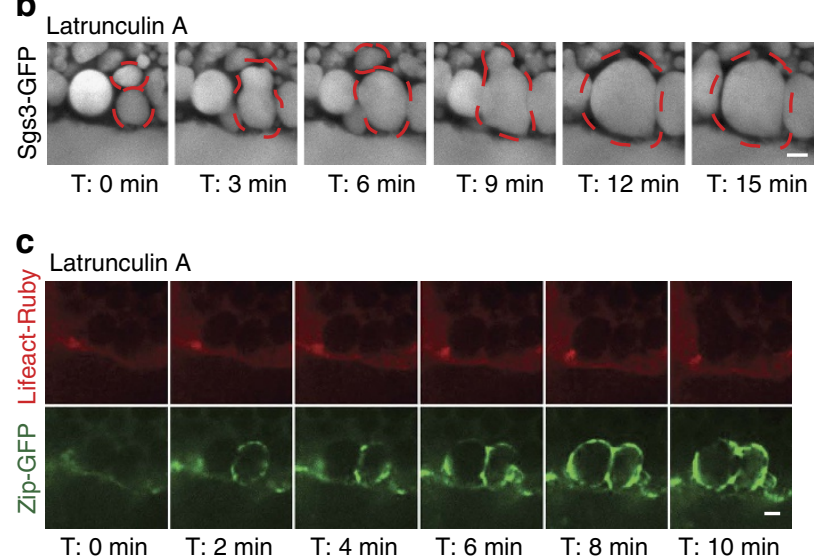

Figure 3 | Actin is required for secretion and inhibition of compound vesicle fusion. (a) Secreting glands expressing Sgs3-GFP (white) were treated with latrunculin $A$ and individual granule fusion events were imaged in real time. Red boxes denote areas shown as magnified images in $\mathbf{b}$.

(b) Drug treatment resulted in compound fusion events between granules that continue to expand and fail to collapse once fused with the apical membrane. Dashed red lines outline secretory granules undergoing expansion and compound fusion events. A representative times series from three independent experiments is shown. (c) Secreting glands expressing Lifeact-Ruby (red) and Zip-GFP (green) treated with latrunculin A show that actin is no longer recruited to granules that have fused with the apical membrane. A representative time series from four independent experiments is shown. All scale bars, $5 \mu \mathrm{m}$. Complete movies for each time series can be found in the Supplementary Movie section.

observation supports a role for cortical actin acting as a barrier to premature vesicle fusion, as has been proposed for other modes of secretion $^{4-6,45}$. Shortly after this cortical rearrangement, actin begins to expand over the secretory vesicle from the site of PM contact. Previous studies imaging actin (as it is involved in exocytosis) in two dimensions suggest that recruitment occurs simultaneously over all regions of the vesicle ${ }^{46}$. However, our $4 \mathrm{D}$ renderings provide spatial and temporal resolution that has not been seen previously and show that actin recruitment to the secretory vesicle begins at the apical PM. These dynamic changes in actin organization suggest a highly ordered process whereby factors on the PM and vesicle act in concert to remodel actin to mediate secretory vesicle docking/fusion as well as to reform the actomyosin machinery around the fused vesicle. This mode of actin recruitment may provide the continuity with cortical actin required to generate the appropriate forces needed for cargo expulsion/release, especially in cases of large, highly glycosylated cargo, such as mucins.

Actin recruitment to secretory vesicles was preceded by the formation of the fusion pore between the apical PM and the secretory vesicle membrane. In addition, membrane mixing and redistribution of the membrane lipid $\mathrm{PIP}_{2}$ also occurred before F-actin recruitment. As $\mathrm{PIP}_{2}$ is known to mediate actin formation in other systems ${ }^{38,39}$, our results suggest that this membrane a
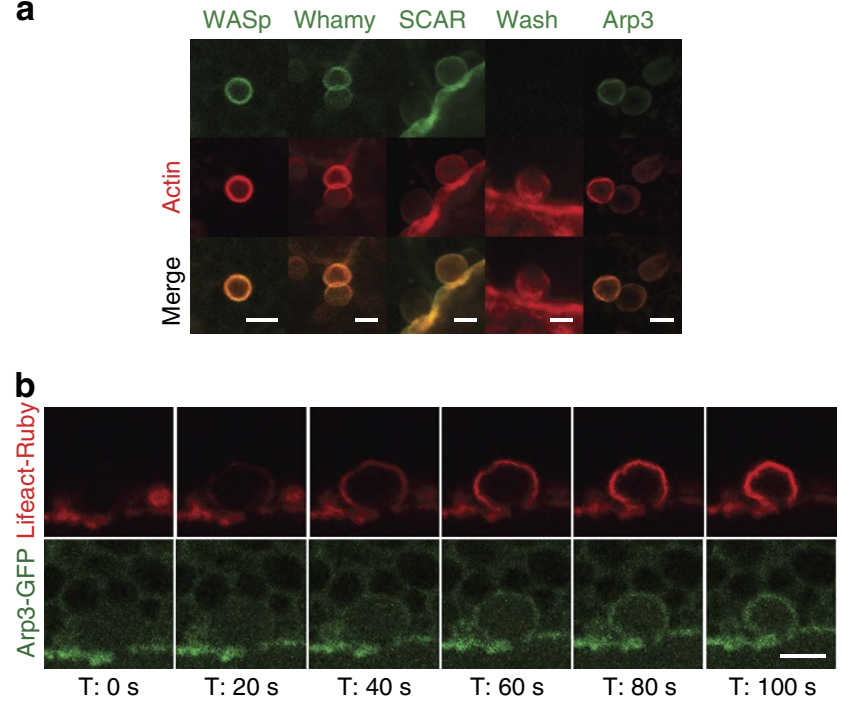

C

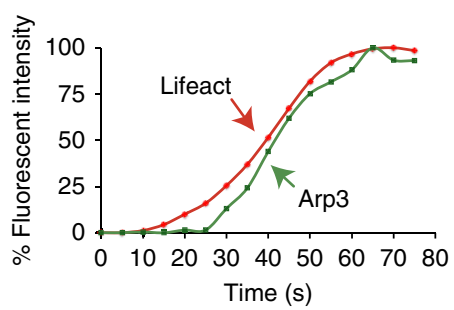

Figure 4 | Arp2/3 and WASp are recruited to granules undergoing secretion. (a) Secreting glands were fixed and stained for Arp3, WASp, Whamy, SCAR or Wash (green) along with phalloidin (red). Merged images are shown in yellow. Representative images from three independent experiments are shown. (b) Secreting glands expressing both Arp3-GFP (green) and Lifeact-Ruby (red) were imaged in real time to show that actin is detected on fused granules before Arp3, suggesting that linear actin first coats fused granules, followed by branched actin formation. A representative time series from 11 independent experiments is shown. (c) Quantification of actin recruitment relative to Arp3 recruitment to fused granules. Representative graph plotting percent fluorescent intensity of each marker as a function of time for an individual secretory event is shown. Quantification was performed on 13 individual secretory events from four independent experiments. Additional graphs are shown in Supplementary Fig. 3b. All scale bars, $5 \mu \mathrm{m}$. Complete movies for each time series can be found in the Supplementary Movie section.

mixing may trigger actin polymerization on secretory granule membranes. Interestingly, actin polymerization appears to occur in two distinct steps, as the recruitment of Lifeact to secretory granules is detected before Arp3 recruitment. As the Arp2/3 complex is known to bind linear actin, this suggests that actin formation on fused secretory granules begins with linear actin formation (detected by Lifeact), which originates from the apical PM. Linear actin is then bound by the Arp $2 / 3$ complex, which catalyses the formation of branched actin. Myosin recruitment occurs after actin is present, as has been seen in cell-based systems ${ }^{12}$. Future work will employ this system to temporally dissect the recruitment of other factors involved in secretion.

The ordered recruitment of linear and branched forms of actin suggests essential roles for each in regulated secretion. However, specific roles for branched actin in regulated secretion were previously unknown. Taking advantage of genetic tools, we directly interrogated the role of branched actin in regulated 
secretion by performing RNAi to the genes that regulate its formation. In vivo knockdown of Arp2 or Arp3, both of which are involved in the synthesis of branched actin, resulted in similar phenotypes, where secretory vesicles fused with the PM but were unable to secrete their cargo and unable to undergo membrane integration. These vesicles grew very large and in some instances, separated from the PM and floated back into the cytoplasm. In addition, in vivo knockdown of the NPF WASp resulted in similar phenotypes, identifying it as an additional regulator of exocytosis in this system. Taken together, our results suggest that branched actin is required to generate the force necessary to complete expulsion of the secretory cargo and integration of vesicular membranes with the PM. In the absence of branched actin, the fused secretory vesicles continue to expand in size, likely due to the hydration and expansion of the highly glycosylated mucinous cargo present within. These results have implications for the importance of branched actin formation in other systems such as the digestive tract, where secretion of large, highly glycosylated/ cross-linked cargo is essential for extracellular matrix formation, microbial interactions and protection of epithelial cell surfaces $^{47,48}$.

Our results also suggest unique roles for linear actin in regulated exocytosis. $\mathrm{LA}$ and $\mathrm{CD}$, which disrupt the formation of both linear and branched actin, resulted in compound fusion of secretory vesicles with one another, which was not seen upon

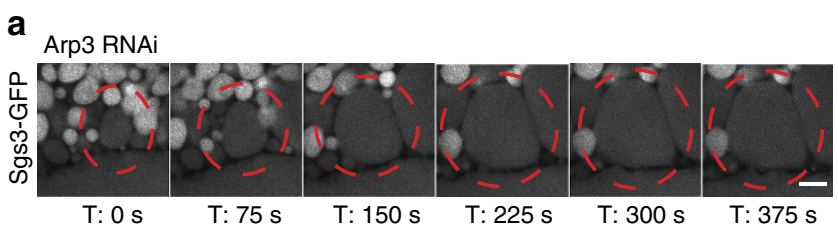

b

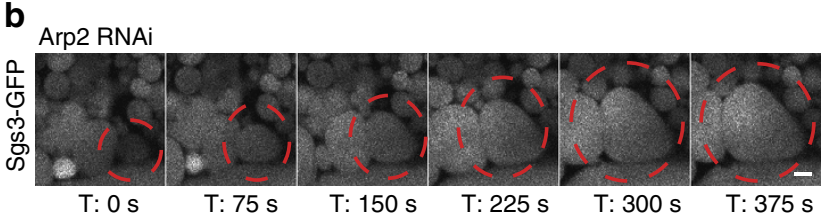

C WASp RnAi

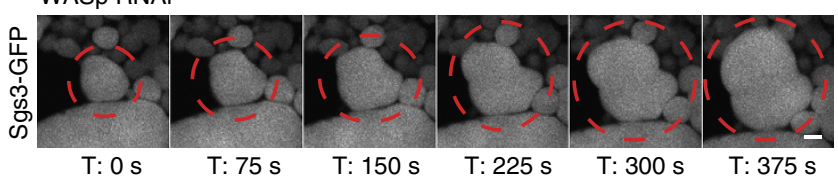

d

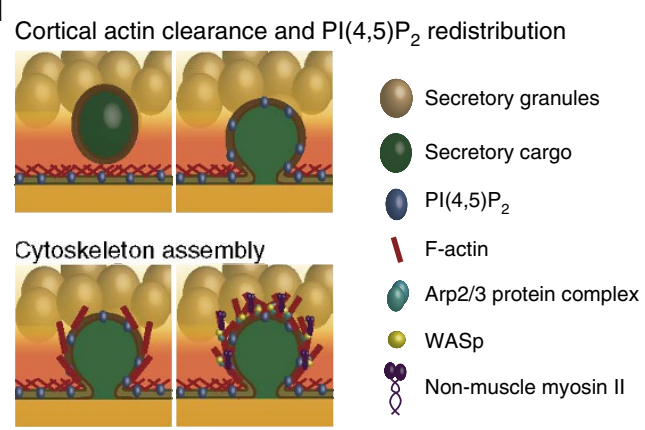

Arp2/3-dependent cargo expulsion and membrane integration

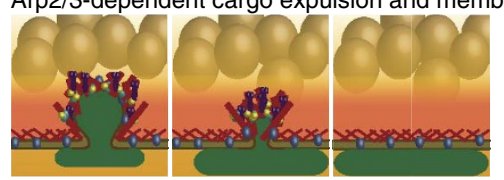

knockdown of Arp2, Arp3 or WASp. This suggests that the linear actin first recruited to the fused secretory vesicle may act as a barrier to prevent the fusion of other secretory vesicles. However, we cannot rule out the possibility that residual branched actin present in our knockdown animals may be able to prevent compound fusion events. Future studies combining fluorescent imaging and electron microscopy of genetically manipulated SGs will aim to identify specific actin structures present on fused secretory granules and how they influence vesicle interactions.

Our study is unique in that we image secretory cargo as a direct indicator of secretion. Previous studies have used visualization of the fusion pore as evidence of cargo secretion ${ }^{8}$. However, in our system, fusion pore formation and cargo expulsion are temporally distinct events, indicating that fusion pore formation alone is not sufficient for secretion of vesicle contents. In addition to the forces mediated by branched actin, other factors may be required for the secretion of large, glycosylated cargos. This system can be used to interrogate other genes that regulate the processing, packaging and release of complex cargos.

In summary, our study highlights coordinated actin clearance and directional recruitment from the PM as well as essential roles for branched actin nucleators in regulated exocytosis in a living, secreting organ. The powerful combination of Drosophila genetics with in vivo and ex vivo imaging allows one to rapidly interrogate the role of factors in many aspects of secretion, including vesicle biogenesis, vesicle movement, fusion with the PM, release of granule cargo and expansion of cargo once in the lumen. Given that many genes and mechanisms involved in secretion are conserved across species, this system can provide insight into the factors that regulate mammalian secretion.

Figure 5 | Arp2/3 and WASp are required for cargo secretion and membrane integration. (a) In vivo RNAi to Arp3 was performed in Drosophila lines that also express Sgs3-GFP (white) and secretion was imaged in real time. Knockdown of Arp3 resulted in granules that fused with the apical membrane but failed to collapse and secrete their contents. A representative time series from three independent experiments is shown. (b) In vivo RNAi to Arp2 was performed in Drosophila lines that also express Sgs3-GFP (white) and secretion was imaged in real time. Loss of Arp2 mimicked the secretion defects seen upon loss of Arp3. A representative time series from three independent experiments is shown. (c) In vivo RNAi to WASp was performed in Drosophila lines that also express Sgs3-GFP (white) and secretion was imaged in real time. Loss of WASp mimicked the secretion defects see upon loss of either Arp2 or Arp3. A representative time series from four independent experiments is shown. All scale bars, $5 \mu \mathrm{m}$. Complete movies for each time series can be found in the Supplementary Movie section. (d) Model of temporal and spatial events occurring during regulated exocytosis in Drosophila SGs. Fully formed secretory granules reside in the secretory cells of third instar larval SGs until secretion is triggered by a pulse of $20 \mathrm{E}$. The early events of secretion involve cortical actin clearance along with fusion pore formation and redistribution of apical $\mathrm{PIP}_{2}$ to the vesicle membranes. The exact order of these events is not known. Following these early events, components of the actin cytoskeleton are assembled on fused granules. First, linear actin is recruited followed by the recruitment of the Arp2/3 protein complex, which forms branched actin structures from existing linear filaments. Actin recruitment occurs in a directional manner originating from the PM. Actin recruitment is followed by recruitment of non-muscle myosin II. Finally, expulsion of cargo from secretory granules and integration of granular membrane with the apical PM is dependent on Arp2-, Arp3- and WASpmediated actin formation. 


\section{Methods}

Fly strains and genetics. The following Drosophila lines were used: Bloomington \#5884 ( $w^{*}$; P\{Sgs3-GFP $\left.\} 2\right)^{32}$, \#5885 ( $w^{*} ;$ P $\{$ Sgs3-GFP 3$)$, \#35544 $\left(y^{1} w^{*} ;\right.$ P $\left.\{U A S-L i f e a c t-G F P\} V I E-260 B\right), \# 35545\left(y^{1} w^{*} ;\right.$ P $\{U A S-L i f e a c t-R u b y\}$ VIE-19A), \#7118 ( $w^{1118}$; P\{UAS-myr-mRFP 1$)$, \#32221 ( $w^{*} ;$ P $\{10 X U A S-I V S$ myr::tdTomato\}attP2), \#39723 ( $w^{*} ;$ P $\{$ UASp-Arp3.GFP $\left.\}\right)^{49}, \# 39693\left(y^{1} w^{*}\right.$ $P\{U A S-P L C \delta$-PH-EGFP $\}$ ), \#58362 ( $w^{*} ;$ P $\left.\{U A S t-L i f e a c t-R F P\} 3\right)$. RNAi lines containing upstream activating sequence (UAS)-inducible inverted repeats (IR) were from the Vienna Drosophila RNAi Center (VDRC) ${ }^{50}$ and were the following stocks: VDRC \#29944 (P\{UAS-Arp2IR), VDRC \#108951 (P\{UAS-Arp3IR), VDRC \#108220 (P\{UAS-WaspIR), VDRC \#60008 $\left(w^{1118} ;\right.$ P $\left\{U A S\right.$-dicer $\left.\left.2^{w+}\right\}\right)$ and VDRC $60009\left(w^{1118} ; P\left\{U A S\right.\right.$-dicer $\left.\left.2^{w+}\right\}\right)$. The wild-type stocks used were either Oregon $R$ or $w^{1118}$ (VDRC \#60000). The Gal4 driver line used in these studies was Bloomington stock \#6978 ( $w^{1118} ; P\{G a w B\} c 135$ ), which drives expression in the Drosophila SG beginning during embryonic stage 15 and continuing through the third instar larval stage. The $c 135-$ Gal4 driver stock was recombined with Sgs3-GFP to generate $c 135-$ Gal4 $>$ Sgs3-GFP. VDRC \#60008, which expresses dicer2 under the control of the UAS promoter, was crossed to VDRC \#108951 and VDRC \#108220 to enhance RNAi-mediated knockdown. VDRC \#60009, which also expresses dicer2, was crossed to VDRC \#29944 to enhance gene knockdown. Sgs3-GFP was recombined with Bloomington \#3554 to generate Sgs3-GFP, UAS-Lifeact-Ruby. Sgs3-GFP was recombined with Bloomington \#3222 1to generate Sgs3-GFP, UASMyr-tdTomato. Bloomington \#3554 was recombined with Flytrap line \#CC01626 to generate Zip-GFP, UAS-Lifeact-Ruby. The c135-Gal4 driver was recombined with Zip-GFP, UAS-Lifeact-Ruby to generate c135-Gal4>Zip-GFP, UAS-LifeactRuby. Bloomington \#3554 was recombined with Bloomington \#39723 to generate UAS-Arp3-GFP, UAS-Lifeact-Ruby. The c135-Gal4 driver was recombined with Bloomington \#58362 to generate c135-Gal4> UAS-Lifeact-RFP. All Drosophila crosses were kept on MM media (KD Medical, Inc.) at $25^{\circ} \mathrm{C}$ unless specified otherwise. Crosses to generate expression of dsRNA were performed using flies from a UAS-IR transgenic line and the c135-Gal4 > Sgs3-GFP or c135-Gal4 > ZipGFP, UAS-Lifeact-Ruby stocks.

Ex vivo SG culture and real-time imaging. SGs from third instar wandering larvae were dissected in Schneider's Drosophila medium (Gibco) and transferred to glass bottom culture dishes (MatTek) containing $50 \mu \mathrm{l}$ media. To immediately image glands that were actively secreting, the majority of the media were then removed from the dish and a $13-\mathrm{mm}, 0.05 \mu \mathrm{m}$, polycarbonate membrane filter (Sterlitech) was gently placed on top of the gland. The media that were removed from the dish were then placed on top of the membrane filter and the sample was subsequently imaged. For imaging sessions longer than $2 \mathrm{~h}, 0.5 \%$ low melt agarose in Schneider's Drosophila medium was layered on top of glands and the sample was subsequently imaged. To induce secretion in non-secreting glands, 20 -hydroxyecdysone (Sigma) was added to glands in glass bottom dishes to a final concentration of $700 \mu \mathrm{M}$ and dishes were placed in a humidified chamber at room temperature for $90 \mathrm{~min}$. The media from these dishes were then removed, a membrane was gently placed on top of the glands and media were added back to the top of the membrane. For experiments involving fluorescent dextran, SGs from non-secreting third instar wandering larvae were dissected and transferred to glass bottom culture dishes containing $50 \mu \mathrm{l}$ media. Alexa Fluor 647, Alexa Fluor 680 or Rhodamine B Dextran (Invitrogen) was subsequently added to the media to a final concentration of $1 \mathrm{mM}$ and then nutated at room temperature for $45 \mathrm{~min}$. 20 -Hydroxyecdysone was then added to a final concentration of $700 \mu \mathrm{M}$ and placed in a humidified chamber at room temperature for $90 \mathrm{~min}$. The media from these dishes were then removed, a membrane was gently placed on top of the glands and media were added back to the top of the membrane. For inhibitor experiments, LA (final concentration $3 \mu \mathrm{M}$ ) or CD (final concentration $10 \mu \mathrm{M}$ ) was added to the media resting on top of the polycarbonate membrane immediately before imaging. All glands were imaged on either a Zeiss LSM 510, Zeiss LSM 700 with a C-Apochromat $\times 63 / 1.2 \mathrm{~W}$ Corr UV-VIS-IR objective or a Nikon A1R + confocal microscope with both CFI Lambda S Apo LWD $\times 40 / 1.15$ numerical aperture (NA) water immersion (WI) and CFI Plan Apo IR $\times 60 / 1.27$ NA WI objectives.

In vivo SG imaging. Wandering third instar larvae were immobilized between two $24 \times 50 \mathrm{~mm}^{2}$ coverslips in sufficient Schneider's media such that the liquid spread evenly across the entire area of the coverslip. Imaging was performed on a Nikon A1R + confocal microscope, with $\times 10$ Plan Apo 0.45 NA or $\times 20$ Plan Apo VC 0.75 NA objective, using the 'keep object in view' function of the advanced tracking module to compensate for $X-Y$ movement. Single confocal sections were acquired every $5 \mathrm{~s}$ to keep glands in the imaging window and a Z-stack was imaged every minute to accommodate movement in the $Z$ plane.

Imaging of prepupae. Prepupae were initially photographed in original MM media vials used to set up crosses. Images were acquired on a Leica MZ 16F fluorescent stereomicroscope with DC 500 camera. Following low-magnification imaging, the prepupae were then transferred to glass bottom culture dishes (MatTek) containing $50 \mu \mathrm{l}$ media. A $13-\mathrm{mm}, 0.05 \mu \mathrm{m}$, polycarbonate membrane filter (Sterlitech) was gently placed on top of the prepupae and used to roll the sample such that SGs were closest to the cover glass. Z-stacks were acquired on a Nikon A1R + confocal microscope with a $\times 20$ Plan Apo VC 0.75 NA objective.

SG fixation and staining. SGs from third instar wandering larvae were dissected in Schneider's Drosophila Medium (Gibco) and immediately transferred to $4 \%$ paraformaldehyde in PBS. Glands were fixed for $20 \mathrm{~min}$ at $4{ }^{\circ} \mathrm{C}$. Samples were then washed three times in PBST (PBS, $0.1 \%$ Triton X-100) and transferred to blocking buffer ( $2 \%$ Goat serum/PBS, $0.1 \%$ Triton X-100) for $1 \mathrm{~h}$ at room temperature on rocker. Samples were then incubated with primary antibodies overnight at $4{ }^{\circ} \mathrm{C}$. Primary antibodies used were mouse anti-WASp (P5E1-Developmental Studies Hybridoma Bank, 1:10), mouse anti-Wash (P3H3-Developmental Studies Hybridoma Bank, 1:20), mouse anti-SCAR (P1C1-Developmental Studies Hybridoma Bank, 1:50), mouse anti-Whamy (P4A8-Developmental Studies Hybridoma Bank, 1:20) ${ }^{23}$ and rabbit anti-Arp3 (the kind gift of Dr W. Theurkauf, 1:500) (ref. 51). Samples were then washed three times for 15 min each in PBST and incubated with secondary antibodies for $3 \mathrm{~h}$ at room temperature on a rocker. Alexa Fluor 488- or Alexa Fluor 647-conjugated secondary antibodies (Invitrogen, 1:50) and TRITC-conjugated phalloidin (Sigma, 1:100) were used. Samples were then washed three times for 15 min each in PBST, mounted in aqueous mounting medium (Electron Microscopy Sciences) and imaged using a Zeiss LSM 700 confocal microscope.

Quantitative RT-PCR. Quantitative RT-PCR was used to determine efficiency of gene silencing when RNAi was induced. cDNA prepared from SGs from third instar wandering larvae of c135-Gal4,Sgs3-GFP > IR crosses or controls (c135Gal4,Sgs3-GFP > VDRC\#60000 or OregonR) was used in qPCR reactions. Briefly, RNA was isolated using the RNAqueous-Micro Total RNA Isolation Kit (Ambion) cDNA synthesis was performed using iScript cDNA Synthesis Kit (Bio-Rad). PCR primers (Supplementary Fig. 7d) were designed using Beacon Designer software (Bio-Rad). QRT-PCR was performed on a CFX96 real-time PCR thermocycler (Bio-Rad) using the SYBR-Green PCR Master Mix (Bio-Rad). RNA levels were normalized to $18 \mathrm{~S}$ rRNA. Values represent mean value \pm s.e.m. Significance values were calculated using two-tailed Student's $t$-test.

Statistical analyses. Number of replicates used for each analysis is specified in figure legends. To measure percent fluorescent intensity for Lifeact/Dextran, Lifeact/PLC $\delta$-PH, Lifeact/Zip or Lifeact/Arp3 experiments, Nikon Elements software was used. A rectangular or oval region of interest (ROI) was drawn around individual secreting granules and the Time Measurement analysis tool was used to measure average fluorescent intensity within the ROI for each channel. Values were exported to Microsoft Excel and percent fluorescent intensity was calculated. The percent fluorescent intensity of each channel at each time point was calculated as: $\%$ fluorescent intensity $=($ Fluorescent intensity-Fluorescent intensity of $\left.\mathrm{T}_{0}\right) /\left(\right.$ Fluorescent intensity of $T_{\max }$-Fluorescent intensity of $\left.T_{0}\right) \times 100 . T_{0}$ is the time frame at which one of the two channels being measured begins to continually increase in fluorescent intensity. $T_{\max }$ is the maximum fluorescent intensity value reached for each respective channel being measured. Each graph was plotted from $T_{0}$ until at least two time point beyond $T_{\max }$ for each channel. Percent fluorescent intensity values that were negative due to fluctuations in intensity values as a result of granule movement were set to 0 . Quantification was only performed on granules that did not move within or through the imaging plane during the entire process of secretion. To determine the average time difference in the detection of each component in the pairwise comparisons, we calculated the time between $T_{0}$ and when fluorescent intensity of the second component began to continually increase and was at least $1 \%$ higher than the previous time point. Measurements were performed on 11 granules for Lifeact/Dextran, 12 granules for Lifeact/PLC $\delta$-PH, 11 granules for Lifeact/Zip and 13 granules for Lifeact/Arp3. Averages were calculated for each pair and are expressed as mean values \pm s.d. To measure fold change in size of secreting Sgs3-GFP granules, Fiji (http://fiji.sc/Fiji) was used to outline and measure the area of individual secreting granules as a function of time after fusion. Granule fusion with the apical membrane correlates with a change in fluorescent intensity of the fusing granule. Time $0 \mathrm{~s}$ represents the frame immediately before a change in fluorescent intensity of a given granule is detected. Values were exported to Microsoft Excel and fold change in granule size was calculated. Graphs represent mean values of all granules measured \pm s.d. Quantification was only performed on granules where fusion with the apical membrane could be detected and could be clearly visualized throughout the time frame of analysis. No statistical method was used to predetermine sample size.

Image analysis. Microscopy images were opened in Fiji and individual frames were exported as still images or Movies. Movies were subsequently formatted using Apple Final Cut Pro, Adobe Media Encoder or Apple Quicktime software. Imaris Bitplane software was used to perform surface rendering of $4 \mathrm{D}$ Lifeact experiments. Adobe Illustrator was used to generate figures from individual still images. 


\section{References}

1. Masedunskas, A., Porat-Shliom, N. \& Weigert, R. Regulated exocytosis: novel insights from intravital microscopy. Traffic 13, 627-634 (2012).

2. Nightingale, T. D., Cutler, D. F. \& Cramer, L. P. Actin coats and rings promote regulated exocytosis. Trends Cell Biol. 22, 329-337 (2012).

3. Porat-Shliom, N., Milberg, O., Masedunskas, A. \& Weigert, R. Multiple roles for the actin cytoskeleton during regulated exocytosis. Cell Mol. Life Sci. 70, 2099-2121 (2013).

4. Brown, A. C. et al. Remodelling of cortical actin where lytic granules dock at natural killer cell immune synapses revealed by super-resolution microscopy. PLoS Biol. 9, e1001152 (2011).

5. Giner, D. et al. Real-time dynamics of the F-actin cytoskeleton during secretion from chromaffin cells. J. Cell Sci. 118, 2871-2880 (2005).

6. Trifaro, J. M., Gasman, S. \& Gutierrez, L. M. Cytoskeletal control of vesicle transport and exocytosis in chromaffin cells. Acta Physiol. (Oxf) 192, 165-172 (2008)

7. Sokac, A. M., Co, C., Taunton, J. \& Bement, W. Cdc42-dependent actin polymerization during compensatory endocytosis in Xenopus eggs. Nat. Cell Biol. 5, 727-732 (2003).

8. Sokac, A. M., Schietroma, C., Gundersen, C. B. \& Bement, W. M. Myosin-1c couples assembling actin to membranes to drive compensatory endocytosis. Dev. Cell 11, 629-640 (2006).

9. Yu, H. Y. \& Bement, W. M. Control of local actin assembly by membrane fusion-dependent compartment mixing. Nat. Cell Biol. 9, 149-159 (2007)

10. Yu, H. Y. \& Bement, W. M. Multiple myosins are required to coordinate actin assembly with coat compression during compensatory endocytosis. Mol. Biol. Cell 18, 4096-4105 (2007).

11. Jerdeva, G. V. et al. Actin and non-muscle myosin II facilitate apical exocytosis of tear proteins in rabbit lacrimal acinar epithelial cells. J. Cell Sci. 118, 4797-4812 (2005)

12. Miklavc, P. et al. Actin depolymerisation and crosslinking join forces with myosin II to contract actin coats on fused secretory vesicles. J. Cell Sci. 128, 1193-1203 (2015)

13. Miklavc, P. et al. Actin coating and compression of fused secretory vesicles are essential for surfactant secretion--a role for Rho, formins and myosin II. J. Cell Sci. 125, 2765-2774 (2012).

14. Nightingale, T. D. et al. Actomyosin II contractility expels von Willebrand factor from Weibel-Palade bodies during exocytosis. J. Cell Biol. 194, 613-629 (2011).

15. Larina, O. et al. Dynamic regulation of the large exocytotic fusion pore in pancreatic acinar cells. Mol. Biol. Cell 18, 3502-3511 (2007).

16. Masedunskas, A. et al. Role for the actomyosin complex in regulated exocytosis revealed by intravital microscopy. Proc. Natl Acad. Sci. USA 108, 13552-13557 (2011).

17. Faix, J. \& Grosse, R. Staying in shape with formins. Dev. Cell 10, 693-706 (2006).

18. Gasman, S., Chasserot-Golaz, S., Hubert, P., Aunis, D. \& Bader, M. F. Identification of a potential effector pathway for the trimeric Go protein associated with secretory granules. Go stimulates a granule-bound phosphatidylinositol 4-kinase by activating RhoA in chromaffin cells. J. Biol. Chem. 273, 16913-16920 (1998).

19. Goode, B. L. \& Eck, M. J. Mechanism and function of formins in the control of actin assembly. Annu. Rev. Biochem. 76, 593-627 (2007).

20. Goley, E. D. \& Welch, M. D. The ARP2/3 complex: an actin nucleator comes of age. Nat. Rev. Mol. Cell Biol. 7, 713-726 (2006).

21. Pollard, T. D. \& Cooper, J. A. Actin, a central player in cell shape and movement. Science 326, 1208-1212 (2009).

22. Firat-Karalar, E. N. \& Welch, M. D. New mechanisms and functions of actin nucleation. Curr. Opin. Cell Biol. 23, 4-13 (2011).

23. Rodriguez-Mesa, E., Abreu-Blanco, M. T., Rosales-Nieves, A. E. \& Parkhurst, S. M. Developmental expression of Drosophila Wiskott-Aldrich Syndrome family proteins. Dev. Dyn. 241, 608-626 (2012)

24. Rotty, J. D., Wu, C. \& Bear, J. E. New insights into the regulation and cellular functions of the ARP2/3 complex. Nat. Rev. Mol. Cell Biol. 14, 7-12 (2013).

25. Blanchoin, L. et al. Direct observation of dendritic actin filament networks nucleated by Arp2/3 complex and WASP/Scar proteins. Nature 404, 1007-1011 (2000).

26. Mullins, R. D., Heuser, J. A. \& Pollard, T. D. The interaction of Arp2/3 complex with actin: nucleation, high affinity pointed end capping, and formation of branching networks of filaments. Proc. Natl Acad. Sci. USA 95, 6181-6186 (1998).

27. Uenishi, E. et al. Actin dynamics regulated by the balance of neuronal WiskottAldrich syndrome protein (N-WASP) and cofilin activities determines the biphasic response of glucose-induced insulin secretion. J. Biol. Chem. 288, 25851-25864 (2013).
28. Gorr, S. U., Venkatesh, S. G. \& Darling, D. S. Parotid secretory granules: crossroads of secretory pathways and protein storage. J. Dent. Res. 84, 500-509 (2005).

29. Burgess, J. et al. Type II phosphatidylinositol 4-kinase regulates trafficking of secretory granule proteins in Drosophila. Development 139, 3040-3050 (2012).

30. Burgess, J. et al. AP-1 and clathrin are essential for secretory granule biogenesis in Drosophila. Mol. Biol. Cell 22, 2094-2105 (2011).

31. Ashburner, M. \& Berendes, H. D. Puffing of polytene chromosomes (Academic Press, 1978).

32. Biyasheva, A., Do, T. V., Lu, Y., Vaskova, M. \& Andres, A. J. Glue secretion in the Drosophila salivary gland: a model for steroid-regulated exocytosis. Dev. Biol. 231, 234-251 (2001).

33. Boyd, M. \& Ashburner, M. The hormonal control of salivary gland secretion in Drosophila melanogaster: studies in vitro. J. Insect Physiol. 23, 517-523 (1977).

34. Hatan, M., Shinder, V., Israeli, D., Schnorrer, F. \& Volk, T. The Drosophila blood brain barrier is maintained by GPCR-dependent dynamic actin structures. J. Cell Biol. 192, 307-319 (2011).

35. Riedl, J. et al. Lifeact: a versatile marker to visualize F-actin. Nat. Methods $\mathbf{5}$, 605-607 (2008)

36. Pfeiffer, I., Petronis, S., Koper, I., Kasemo, B. \& Zach, M. Vesicle adsorption and phospholipid bilayer formation on topographically and chemically nanostructured surfaces. J. Phys. Chem. B 114, 4623-4631 (2010).

37. Shewan, A., Eastburn, D. J. \& Mostov, K. Phosphoinositides in cell architecture Cold Spring Harbor Perspect. Biol. 3, a004796 (2011).

38. Bothe, I., Deng, S. \& Baylies, M. PI $(4,5)$ P2 regulates myoblast fusion through Arp2/3 regulator localization at the fusion site. Development 141, 2289-2301 (2014).

39. Higgs, H. N. \& Pollard, T. D. Activation by Cdc42 and PIP(2) of WiskottAldrich syndrome protein (WASp) stimulates actin nucleation by Arp2/3 complex. J. Cell Biol. 150, 1311-1320 (2000).

40. Verstreken, P. et al. Tweek, an evolutionarily conserved protein, is required for synaptic vesicle recycling. Neuron 63, 203-215 (2009).

41. Buszczak, M. et al. The carnegie protein trap library: a versatile tool for Drosophila developmental studies. Genetics 175, 1505-1531 (2007).

42. Lee, D. M. \& Harris, T. J. An Arf-GEF regulates antagonism between endocytosis and the cytoskeleton for Drosophila blastoderm development. Curr. Biol. 23, 2110-2120 (2013).

43. Birchenough, G. M., Johansson, M. E., Gustafsson, J. K., Bergstrom, J. H. \& Hansson, G. C. New developments in goblet cell mucus secretion and function. Mucosal Immunol. 8, 712-719 (2015).

44. Nilsson, H. E. et al. Intestinal MUC2 mucin supramolecular topology by packing and release resting on D3 domain assembly. J. Mol. Biol. 426, 2567-2579 (2014).

45. Gutierrez, L. M. New insights into the role of the cortical cytoskeleton in exocytosis from neuroendocrine cells. Int. Rev. Cell Mol. Biol. 295, 109-137 (2012).

46. Jang, Y., Soekmadji, C., Mitchell, J. M., Thomas, W. G. \& Thorn, P. Real-time measurement of F-actin remodelling during exocytosis using Lifeact-EGFP transgenic animals. PLoS ONE 7, e39815 (2012).

47. Ambort, D. et al. Calcium and pH-dependent packing and release of the gel-forming MUC2 mucin. Proc. Natl Acad. Sci. USA 109, 5645-5650 (2012).

48. Schutte, A. et al. Microbial-induced meprin beta cleavage in MUC2 mucin and a functional CFTR channel are required to release anchored small intestinal mucus. Proc. Natl Acad. Sci. USA 111, 12396-12401 (2014).

49. Rajan, A., Tien, A. C., Haueter, C. M., Schulze, K. L. \& Bellen, H. J. The Arp2/3 complex and WASp are required for apical trafficking of Delta into microvilli during cell fate specification of sensory organ precursors. Nat Cell Biol. 11, 815-824 (2009).

50. Dietzl, G. et al. A genome-wide transgenic RNAi library for conditional gene inactivation in Drosophila. Nature 448, 151-156 (2007).

51. Stevenson, V., Hudson, A., Cooley, L. \& Theurkauf, W. E. Arp2/3-dependent pseudocleavage furrow assembly in syncytial Drosophila embryos. Curr. Biol. $12,705-711(2002)$

\section{Acknowledgements}

We thank our colleagues for many helpful discussions. We thank Jia Guo for technical assistance. We also thank the Vienna Drosophila RNAi Center, the Bloomington Stock Center and the Developmental Studies Hybridoma Bank for fly stocks and other reagents. This work was supported by the Intramural Research Program of the NIDCR National Institutes of Health (Z01-DE-000713 to K.G.T.H.).

\section{Author contributions}

D.T.T. and K.G.T.H. designed and planned the research with input from R.W. and A. M.; D.T.T. performed the experiments; D.T.T., K.G.T.H., R.W. and A.M. analysed 
and discussed the data; K.G.T.H. and D.T.T. wrote the paper with input from A.M. and R.W.

\section{Additional information}

Supplementary Information accompanies this paper at http://www.nature.com/ naturecommunications

Competing financial interests: The authors declare no competing financial interests.

Reprints and permission information is available online at http://npg.nature.com/ reprintsandpermissions/
How to cite this article: Tran, D. T. et al. Arp2/3-mediated F-actin formation controls regulated exocytosis in vivo. Nat. Commun. 6:10098 doi: 10.1038/ncomms10098 (2015).

cc (i) This work is licensed under a Creative Commons Attribution 4.0 International License. The images or other third party material in this article are included in the article's Creative Commons license, unless indicated otherwise in the credit line; if the material is not included under the Creative Commons license, users will need to obtain permission from the license holder to reproduce the material. To view a copy of this license, visit http://creativecommons.org/licenses/by/4.0/ 